\title{
Participación estudiantil en escuelas chilenas con buena y mala convivencia escolar
}

\author{
Students Participation in Chilean Schools \\ with Good and Poor School Climate
}

\author{
Paula Ascorra, Verónica López, \& Carolina Urbina \\ Pontificia Universidad Católica de Valparaíso, Valparaíso, Chile
}

\begin{abstract}
Resumen: La participación escolar es una deuda pendiente en América Latina que dificulta el avance hacia sociedades democráticas. Este estudio buscó comprender la participación escolar, desde las voces de los estudiantes, en dos escuelas con alta calidad de convivencia escolar y dos escuelas con baja calidad de la convivencia escolar, seleccionadas según un índice creado a partir de encuestas a estudiantes. Con base en un diseño cualitativo, se realizaron entrevistas semiestructuradas grupales a 70 estudiantes de octavo año básico. Los resultados indican que en todas las escuelas la participación de los estudiantes es tutelada e instrumentalmente dirigida por los adultos de la escuela, heteronormativa, selectiva e inestable. Las diferencias entre las escuelas con alta y baja calidad de convivencia escolar es que las primeras limitan la participación no convencional de los estudiantes ligada a la movilización estudiantil extraescuela, y amenazan a los estudiantes con intereses políticos. Se discute la constitución de una subjetividad estudiantil pasiva y dependiente al interior del establecimiento educacional, y activa y comprometida al exterior del mismo, así como la noción misma de convivencia escolar, que pareciera ser de carácter disciplinar.
\end{abstract}

Palabras clave: participación, escuela, estudiantes, convivencia escolar.

\begin{abstract}
School participation is a debt in Latin America that hinders progress towards democratic societies. This study sought to understand school participation, from the voices of students in two schools with high quality school climate and two schools with low quality school climate, selected according to an index created from student surveys. Using a qualitative design, group semi-structured interviews with 70 students from eighth grade were performed. The results indicate that in all schools the participation of students is instrumentally supervised and directed by adults, and that school participation is heteronormative, selective and unstable. The differences between schools with high and low quality school life is that the former limits unconventional student participation linked to extra-school student mobilization, and threaten students who express political interests. We discuss the construction of passive and dependent student subjectivity within the schools, in contrast with an active and committed subjectivity outside the school premises. We also discuss the very notion of school life, which seems to be understood as discipline.
\end{abstract}

Keywords: participation, school, students, school climate.

Este artículo de investigación es parte del proyecto "Convivencia escolar en escuelas municipales vulnerables y no selectivas: relaciones positivas entre rendimiento y convivencia" (Fondecyt \#1140806) y del proyecto "El agenciamiento colectivo en los procesos de mejora educativa", (Fondecyt postdoctoral \#3140204), financiados por el Fondo Nacional de Desarrollo Científico y Tecnológico de Chile.

Contacto: P. Ascorra. Av. El Bosque 1290, Viña del Mar, Región de Valparaíso, Chile. Correo electrónico: paula.ascorra@pucv.cl

Cómo citar: Ascorra, P., López, V., \& Urbina, C. (2016). Participación estudiantil en escuelas chilenas con buena y mala convivencia escolar. Revista de Psicología, 25(2), 1-18.

http://dx.doi.org/10.5354/0719-0581.2016.44686 


\section{Introducción}

El ideario declarado por la Organización de las Naciones Unidas para la Educación, la Ciencia y la Cultura (Unesco, 2000) de instalar una educación para la paz se materializó en el compromiso adoptado por ministerios de educación de diferentes países del mundo, el que consistía en promover formas de vida conjuntas en un marco de respeto a la diversidad, participación y desarrollo de valores democráticos. Del mismo modo, la Convención sobre los Derechos del Niño, aprobada por Naciones Unidas en 1989, reconoce el derecho a la participación del niño en diferentes ámbitos de su vida cotidiana (United Nations Children's Fund, Unicef, 1990).

En Chile, el Ministerio de Educación (Mineduc) y el Ministerio Secretaría General de Gobierno (MSGG) han promovido una política de participación y de convivencia escolar que se traslapan en muchos aspectos. La estrategia ha apuntado hacia una política de transversalidad que promueve tanto el desarrollo de la participación como de la convivencia escolar, asociadas a actividades curriculares. Para ello, se han entregado una serie de cuerpos normativos, entre los que destacan: la "Política de transversalidad" (MSGG, 2001); la "Política de convivencia escolar" (Mineduc, 2002, 2003); "Metodologías de trabajo para el mejoramiento de la convivencia escolar" (Mineduc, 2005); "Políticas de participación para padres, madres y apoderados" (Mineduc, 2002); la "Política de participación en los consejos escolares" (Mineduc, 2005); la "Nueva política de convivencia escolar" (Mineduc, 2011); la "Ley sobre violencia escolar" (Mineduc, 2011); y la reformulación a la Política de convivencia escolar 2015-2018 (Mineduc, 2015).

Dado que existe un traslape en la promoción de la convivencia y la participación escolar, el presente estudio se propone comprender la participación de estudiantes de primaria en escuelas que presentan una alta y una baja calidad de la convivencia escolar. Hipotetizamos que aquellas escuelas que presentan una mejor calidad de su convivencia escolar, presentarán también una mayor participación. Se ha querido profundizar en las voces de los estudiantes, ya que su desconocimiento y silenciamiento es totalmente contrario a la concepción democrática y participativa que diversos ministerios quieren instalar, en que el estudiante es un sujeto de pleno derecho (San Fabián Maroto, 2008).

\section{Participación escolar}

La participación escolar es un concepto de larga data en la investigación en educación. Está fuertemente asociado al concepto de participación ciudadana, en tanto se espera que en la escuela los estudiantes puedan realizar el ejercicio de la ciudadanía, a través de su involucramiento activo en los procesos escolares y también a través de la convivencia escolar (Martínez, Silva, Morandé, \& Canales, 2010; Perliger, Canetti-Nisim, \& Pedahzur, 2006; Rodríguez Rodríguez, 2008; Schulz \& Fraillon, 2012).

La participación activa de los estudiantes en la construcción de sus propios aprendizajes y normas que posibilitan una adecuada y enriquecida convivencia escolar entra fuertemente en tensión con la cultura escolar que se ha caracterizado como autoritaria (Assaél, Edwards, López, \& Adduard, 1989; Berg \& Cornell, 2016) y adultocéntrica (Dávila, 2003; Dávila León, Ghiardo Soto, \& Medrano Soto, 2005). De allí, que algunos investigadores hayan optado por aplicar el concepto de niveles de participación ciudadana al ámbito de la participación escolar, utilizando la metáfora de la escalera de la participación. Este concepto fue inicialmente desarrollado por Arntsein (1969) 
como una metáfora para evaluar los distintos grados de participación ciudadana adulta. Los niveles más altos de la escalera (colaboración, delegación de poder y control ciudadano) representan un mayor grado de poder de la ciudadanía. Los peldaños intermedios (información, consulta) corresponden a un nivel de formulismo; y los peldaños inferiores (manipulación, terapia) se consideran modelos de participación engañosa o pseudo participación, en tanto no generan ni permiten una verdadera participación ciudadana.

Hart (1993) elaboró una versión adaptada para Unicef de la escalera de Arnstein (1969) para clasificar las formas y niveles de participación infantil y juvenil. En esta versión, la población adulta es la que ostenta el poder. El modelo propone una escalera con dos tramos: la no-participación (manipulación o engaño; decoración; participación simbólica) y la participación (de menor a mayor participación: información; consulta e información; iniciado por personas adultas, con decisión infantil; iniciado y dirigido por niñas y niños; iniciado por niñas y niños, con decisiones compartidas con las y los adultos). Mientras que la no-participación construye al niño como sujeto pasivo y carente de opinión y decisión sobre su vida, los niveles de participación suponen que los niños y niñas pueden asumir roles progresivamente más activos en las situaciones de vida que los afectan. Así, en el último peldaño de mayor participación activa, los niños toman decisiones conjuntamente con los adultos.

Los niveles de participación infantil de Hart $(1993,1997)$ han sido considerados por muchos investigadores como un modelo apropiado para estudiar la participación en el ámbito escolar (Tonon, 2012; Wetzelhütter $\&$ Bacher, 2015). En Chile, este modelo fue adaptado por Reca y López (2001) y por
Guzmán, Hojman y López (2002) para estudiar la participación organizada de los padres en el sistema escolar chileno. Se realizó una investigación teórica (Reca \& López, 2001) sobre la participación de los padres en consejos escolares a nivel internacional, y un estudio empírico (Guzmán et al., 2002) sobre la participación de los padres en los centros de padres y apoderados. De esto se concluyó que el nivel de participación de los padres en los centros de padres y apoderados se podía ubicar en el peldaño de la información y consulta, y que la figura de los consejos escolares podría permitir niveles de participación mayor, que facilitaran el ejercicio de la participación ciudadana en los padres y apoderados del sistema escolar chileno. Aun cuando, posterior a esta publicación, se creó en Chile el consejo escolar como órgano de gobierno en los establecimientos escolares, el carácter consultivo que se atribuyó a este -pudiendo ser deliberativo solo bajo autorización del sostenedor- ha limitado significativamente su potencial como vehículo de gobernanza que permitiera la participación ciudadana de padres/apoderados y de estudiantes en la escuela (De la Fuente \& Raczynski, 2010), y un rol de los padres como agentes activos de los procesos de mejoramiento escolar (Montecinos, Sisto, \& Ahumada, 2010).

\section{Participación de los estudiantes chilenos}

Las investigaciones nacionales concuerdan en que hay una gran deuda en la participación efectiva que tienen los alumnos dentro de la escuela, existiendo la necesidad por parte de estos de mayores espacios de participación deliberativa dentro de los establecimientos educativos (Prieto, 2001; Instituto de Ciencia Política, Pontificia Universidad Católica de Chile, 2016). De acuerdo con Muñoz (2011), la participación en el espacio de la escuela chilena ha quedado reducida a una participación 
clientelista, donde -congruentemente con el paradigma neoliberal- los consejos escolares, los centros de padres y los centros de alumnos ejercen su participación en términos de gestionar recursos para financiar mejoras para la escuela.

La participación clientelista no puede atribuirse únicamente a intencionalidades individuales ligadas, por ejemplo, al autoritarismo pedagógico. Más bien, obedecen a racionalidades propias de dispositivos técnico-instrumentales que caracterizaron la racionalidad de las empresas privadas en las décadas de 1940 a 1980, en que los ciudadanos pueden participar, pero bajo propuestas metodológicas construidas en forma externa a la comunidad en que serán instaladas, y que requieren de una autoridad-experta para materializarse (Anderson, 2002; Heras, De la Riestra, \& Burin, 2010).

Para Anderson (2002), los modelos participativos que han sido aplicados a la educación no se traducen en formas de participación auténticas, ya que la mayoría de estos modelos de administración no se usa para crear o desafiar objetivos, sino para que los individuos asuman valores y objetivos preexistentes.

Ahora bien, este tipo de participación difiere sustancialmente de otras formas de participación estudiantil, como las que realizan en los movimientos por los derechos sociales, entre ellos el de educación gratuita y de calidad (Bellei, Contreras, \& Valenzuela, 2010; Bellei, 2016). Al respecto Fleet (2011), tras realizar una lectura sociológica sobre el movimiento estudiantil chileno, concluye que se trata de un movimiento arraigado en la nueva clase media, que es expresión de una crisis de legitimidad y, a la vez, un rechazo activo a las formas actuales de distribución del poder. El autor concluye que la crítica a la distribución del poder a través de la educación "es una plataforma para el planteamiento de un potencial proyecto alternativo de sociedad" (p. 99).

La participación activa y organizada de los estudiantes en el movimiento estudiantil, expresada a través de su involucramiento en las acciones de paros y tomas de los establecimientos escolares, y marchas en las calles, podría considerarse una participación auténtica. Esto debido a que las acciones tienen un fuerte componente político que busca desestructurar y transformar las bases del ordenamiento social, y a que muchas veces no coinciden con los intereses o las voluntades de los adultos en la escuela. Siguiendo el modelo de Hart (1993), se trataría de una participación en el nivel de iniciado y dirigido por niñas y niños, sin decisiones compartidas por las y los adultos.

\section{Participación y convivencia escolar: una discusión pendiente}

La relación entre participación y convivencia escolar tiene una larga data en Iberoamérica. De acuerdo con Rodino (2013), la instalación de una cultura escolar para la paz en América Latina tuvo por objetivo la reconstrucción de la democracia, en un continente fuertemente violentado. No debemos olvidar la presencia de dictaduras militares en Chile, Argentina, Bolivia, Brasil, Uruguay y Paraguay, durante los 70 y 80, así como procesos de violencia e insurrección en El Salvador, Colombia, México y Nicaragua. En este contexto de violencia, el ideario de una convivencia escolar pacífica y participativa otorgaba las garantías para que niños y jóvenes ejercieran sus derechos fundamentales, desarrollaran capacidades de cohesión y participación para una ciudadanía democrática (Rodino, 2013).

El constructo utilizado por el Mineduc (2014) sobre clima de convivencia escolar tiene sus antecedentes en el desarrollo de dos conceptos distintos: school climate de 
origen anglosajón (en adelante clima escolar) y convivencia escolar (de origen latino).

El clima escolar es un constructo multidimensional que hace referencia a la calidad de las relaciones y el ambiente para el aprendizaje (Godás Otero, Santos Rego, \& Lorenzo Moledo, 2008; Wang \& Degol, 2016), siendo su principal dimensión la académica (Thapa, Cohen, Guffey, \& Higgins-D'Alessandro, 2013). Si bien no existe acuerdo amplio respecto de cuáles son las dimensiones que deberían ser evaluadas en el clima escolar, las revisiones más recientes concuerdan en que serían cuatro: seguridad, académica, comunidad y medio ambiente institucional (Wang \& Degol, 2016; Thapa et al., 2013; Cohen, McCabe, Michelli, \& Pickeral, 2009). La seguridad refiere al manejo del bullying y la violencia, y a la existencia de normas, justas claras y conocidas. La dimensión académica hace alusión a los procesos de enseñanza-aprendizaje con apoyo de profesores a estudiantes, al desarrollo de carrera de los profesores y al liderazgo del equipo directivo. La dimensión comunidad refiere a calidad de los vínculos entre estudiantes y entre estos y profesores, la participación, el respeto a la diversidad y a la vinculación de la escuela con la comunidad. El medio ambiente institucional refiere al estado y mantención de una infraestructura adecuada (limpieza, luminosidad, etc.); a la disponibilidad de recursos; y a la estructura entendida como tamaño de la escuela, relación estudiantes/profesores, entre otros.

Por su parte, el constructo de convivencia escolar, usado ampliamente en Latinoamérica, tiene un origen histórico y político distinto al clima escolar. De hecho, no existe una traducción de convivencia escolar al inglés. De acuerdo a Carbajal Padilla (2013), el constructo se utilizó por primera vez en 1900 para caracterizar la Edad de Oro española, época en que judíos, musulmanes y católicos convivían pacíficamente en el mismo territorio. Desde el comienzo el constructo se orientó a la organización de una forma de vida pacífica que atendiera la diferencia. De acuerdo con Ortega-Ruiz (2013), el término hace referencia a la interrelación permanente entre grupos sociales diferentes (ej. mestizos, indoamericanos, mulatos, etc.) en un contexto de creciente preocupación, por oleadas de inmigrantes en Europa que comienzan en la década de los 80 y que se acentúan en nuestros días, y por procesos dictatoriales, de insurrecciones $\mathrm{y}$ guerra civiles en América Latina que aún no consiguen la plena paz del continente.

Para el caso de Chile, en 1980 se instala una nueva constitución política que no reconoce el derecho a la participación. Entre los años 1973 y 1983 se proscriben los centros de padres y centros de alumnos. Recién a partir de 1984, se reinstala el funcionamiento de estos organismos colegiados, reduciendo su ámbito de acción a tareas muy precisas (Muñoz, 2011). La dictadura militar abandona el gobierno con la aprobación de la Ley Orgánica Constitucional de Enseñanza (LOCE, 1990), en la que no se reconoce la participación de los diferentes actores escolares en la construcción del proyecto educativo.

Con la llegada de la democracia en 1990, surgen diversas iniciativas para promover la participación en la escuela. Se comienza a concebir la escuela como un espacio indispensable para el ejercicio de la democratización, la integración y la cohesión de los ciudadanos. Con la aprobación del decreto 524 se estipulan las directrices que establecen el tipo de organización y las funciones de los centros de alumnos. Se propone como finalidad de dichos centros representar y servir a los estudiantes sin contravenir los propósitos del establecimiento educacional ni las normas de la organización escolar. Durante el año 
2005, se aprueba la Ley $\mathrm{N}^{\circ} 19.979$ que obliga a todas las escuelas que reciben aportes del Estado a conformar consejos escolares. Estos organismos, que cuentan con la representación de sostenedores, equipo directivo, profesores, estudiantes y apoderados, tienen la función de intercambiar opiniones y propuestas sobre temas relevantes que atañen a todos los integrantes de la comunidad educativa. Otra instancia de participación institucional es el reglamento de convivencia escolar, que debe ser construido de manera participativa por todos los actores escolares (Mineduc, 2002, 2005, 2015).

En Chile, la Agencia de Calidad ha propuesto la denominación de clima de convivencia escolar. Este es un constructo multidimensional compuesto por: clima de respeto, clima organizado y clima de seguridad (Mineduc, 2014). Resulta extremadamente llamativo que el constructo de clima de convivencia escolar implementado en este país no considere la dimensión de mayor relevancia en la evaluación internacional del clima escolar. Nos estamos refiriendo a la dimensión académica, particularmente a la ausencia de dimensiones vinculadas al proceso de enseñanza y aprendizaje $\mathrm{y}$, al apoyo de profesores y directores en su propio desarrollo de carrera y bienes$\operatorname{tar}$ (Thapa et al., 2013).

Atendiendo a la sobreposición que existe en la política educacional chilena en la promoción de la convivencia y la participación escolar, el presente estudio se propone comprender la participación de estudiantes de secundaria en escuelas que presentan una alta y una baja calidad de la convivencia escolar. El supuesto que guía la investigación relaciona la calidad de la convivencia escolar con la participación; es decir, aquellas escuelas que presentan una mejor calidad de su convivencia escolar, presentarán también una mayor participación.

\section{Método}

Esta investigación se realizó desde una perspectiva cualitativa, a partir del desarrollo de entrevistas grupales a estudiantes. Asimismo, este estudio es parte de una investigación mayor cuyo objetivo fue identificar las relaciones entre rendimiento y convivencia escolar, por ello el acceso al campo de estudio estuvo mediado y facilitado debido al desarrollo de la investigación mayor.

\section{Participantes}

Participaron en este estudio 70 estudiantes de $8^{\circ}$ año de educación básica de cuatro escuelas de la comuna de Valparaíso, Región de Valparaíso (Chile).

Estos estudiantes pertenecían a escuelas identificadas con alto y bajo índice de convivencia escolar (ICE). El ICE fue validado previamente por el equipo de investigación (López et al., 2012) y calculado a partir de resultados previos aportados por una batería de instrumentos de autorreporte que fueron administrados a un total de 4.688 estudiantes de $4^{\circ}, 6^{\circ}$ y $8^{\circ}$ básico de 81 escuelas de la región de Valparaíso bajo un diseño representativo y aleatorio bietápico. Dicha batería estuvo compuesta por los siguientes instrumentos: Escala de Agresión y Victimización (Orpinas \& Frankowski, 2001, adaptada por López \& Orpinas, 2012); Escala de Victimización de Profesores a Estudiantes (adaptada de Espinoza, 2006); Inventario Mi Clase (Ascorra \& Cáceres, 2002); Escala de Clima Escolar (Benbenishty \& Astor, 2005); y Escala de Bienestar Social en la Escuela (adaptada de Keyes, 1998).

En consecuencia, se seleccionaron dos escuelas cuyo ICE se ubicaba en el percentil 90 (buena convivencia escolar; Escuela 1 y Escuela 2); y dos cuyo ICE se ubicaba en el percentil 10 (mala convivencia escolar; 
Escuela 3 y Escuela 4). La tabla 1 presenta las características sociodemográficas de las escuelas a las que pertenecían los estudiantes participantes.

\section{Técnicas de producción de información}

Se realizaron entrevistas grupales participativas (Holstein \& Gubrium, 2006) a dos grupos de estudiantes: i) estudiantes sin cargo de representación al interior de sus establecimientos educacionales, y ii) estudiantes que pertenecían al centro de alumnos en cada una de las escuelas en estudio. Los temas abordados en estas entrevistas fueron los espacios de participación y la significación que los estudiantes le asignan a la participación en el ámbito escolar.

Todas las entrevistas fueron realizadas al interior de las escuelas y durante la jornada escolar, grabadas en audio y trascritas para su posterior análisis.

En cada una de las escuelas la entrevista grupal con el centro de alumnos tuvo una duración aproximada de 45 minutos. Asimismo, se realizaron nueve entrevistas grupales de una duración de entre 30 y 45 minutos a alumnos sin cargo de representación. Se realizó un total de 13 entrevistas grupales (ver tabla 2).

\section{Análisis de la información}

Se realizó un análisis de contenido categorial utilizando como referente conceptual la perspectiva bottom-up que recomienda la teoría fundamentada desde los datos (Glaser \& Strauss, 1967). Con la ayuda del software Atlas.ti (Muñoz, 2003) cada investigador realizó una codificación abierta de los datos. En este proceso participaron tres investigadores. Luego, se consensuaron las categorías que presentaron mayores similitudes y se definieron conceptualmente de manera colectiva. Con este nuevo conjunto de categorías, se utilizó como criterio de rigor la triangulación intersubjetiva (Krause, 1995), con lo cual, después del análisis individual, se procedió a consensuar las categorías en el grupo de investigadores, con el objeto de trabajar únicamente con aquellas categorías que fueran conceptual y metodológicamente consistentes y robustas.

Tabla 1

Características de las escuelas

\begin{tabular}{lcccc}
\hline \multicolumn{1}{c}{ Características } & Escuela 1 & Escuela 2 & Escuela 3 & Escuela 4 \\
\hline Dependencia & Municipal & $\begin{array}{c}\text { Particular } \\
\text { subvencionado }\end{array}$ & Municipal & $\begin{array}{c}\text { Particular } \\
\text { subvencionado }\end{array}$ \\
ICE & 0,53 & 0,75 & $-1,72$ & $-2,45$ \\
$\mathrm{~N}^{\mathrm{o}}$ de estudiantes & 222 & 186 & 482 & 270 \\
\hline
\end{tabular}

Nota: ICE = Índice de convivencia escolar.

Tabla 2

Entrevistas a participantes

\begin{tabular}{lcccc}
\hline & Escuela 1 & Escuela 2 & Escuela 3 & Escuela 4 \\
\hline $\mathrm{N}^{\mathrm{o}}$ de entrevistas grupales & 3 & 4 & 3 & 3 \\
$\mathrm{~N}^{\mathrm{o}}$ total de participantes por escuela & 18 & 22 & 16 & 14 \\
\hline
\end{tabular}




\section{Aspectos éticos}

Todos los participantes dieron su asentimiento a participar en la investigación y sus padres firmaron el consentimiento correspondiente. En dicho documento, quedaron establecidos los objetivos de la investigación, el resguardo de la confidencialidad y la voluntariedad de la participación, entre otros aspectos. Dichos documentos fueron revisados y aprobados por el comité de bioética de la institución patrocinadora (Pontificia Universidad Católica de Valparaíso).

\section{Resultados}

\section{Sistema de categorías emergentes}

De acuerdo con el examen realizado, se identificaron cuatro categorías de análisis: participación tutelada, sentido de comunidad, emocionalidad (positiva o negativa) y limitaciones a la participación política.

Participación tutelada. Se refiere a la percepción de participación a través de las regulaciones que proponen profesores y directivos de la escuela. De esta manera, son los adultos de la escuela los que invitan a ciertos grupos de estudiantes a algunas actividades, o bien, instalan procedimientos de participación en la sala de clase. Por ejemplo:

Entrevistador (E): ¿De qué se trata específicamente (Mesa Educativa) y quiénes van?

Alumno (A): ...van representantes de cada curso, y ahí se puede hablar de muchas cosas de la escuela con algunos profesores, por ejemplo una vez hicimos el campeonato de fútbol de la escuela, hicimos también una cuestión de comida saludable y cosas así, yo iba pero después no fui más porque me abu- rrí, después empezaron a ir otros compañeros de acá, pero después también se terminaron aburriendo.

E: ¿Les gusta participar en ese tipo de actividades donde les dan más responsabilidades a los estudiantes como ustedes?

A: Sí, pero no es que todos vayan a participar a esas cosas, eligen a poquitos, dos o tres (Entrevista N ${ }^{\circ}$ 2, Escuela 3, Bajo ICE).

A2: Nosotros arreglamos este patio el año pasado, lo pintamos y quedó súper bonito, eso fue en el taller de acción social, pero empezó a pasar el tiempo y lo abandonaron y ahora está de nuevo todo sucio y con basura.

E: ¿Y se organizaron por decisión de ustedes para hacer eso?

A1: No, por el taller de aseo social (Entrevista $\mathrm{N}^{\circ}$ 1, Escuela 2, Alto ICE).

A: Siempre son los otros los que dan autorización de participar (Entrevista $\mathrm{N}^{\mathrm{o}}$ 2, Escuela 1, Alto ICE).

Sentido de comunidad. Se refiere a la identificación de un sentido colectivo y de pertenencia que los estudiantes identifican necesario para participar y comprometerlos con su escuela. Por ejemplo:

A4: Somos como una familia que se lleva bien y ella nos trata bien como si fuera nuestra mamá (Entrevista $\mathrm{N}^{\circ} 4$, Escuela 2, Alto ICE).

A1: Bien, porque es como sentirse útil, uno se siente más grande porque tiene más responsabilidades y aparte que hacemos cosas para nosotros mismos (Entrevista $\mathrm{N}^{\circ} 3$, Escuela 2, Alto ICE).

Las escuelas que presentan una alta calidad de la convivencia escolar se caracterizan por presentar un mayor sentido de comunidad. La relación entre participación y sentido de comunidad ya ha sido trabajada por Prieto (2001), quien concibe la 
participación como un proceso de comunicación, ejecución y toma de decisiones que permite el encuentro con un otro.

A4: Es que me gusta hacer cosas en esta escuela, porque es mi escuela.

A1: También valoramos que cuando hay un problema todos se unen y lo solucionan; por ejemplo, cuando le robaron una plata a la profe, todos participaron (Entrevista $\mathrm{N}^{\circ}$ 4, Escuela 2, Alto ICE).

A diferencia de estas escuelas, aquellos estudiantes que pertenecen a organizaciones educativas con baja calidad de la convivencia escolar se caracterizan por hacer explícita la ausencia de pertenencia a una comunidad educativa. Es más, se interpreta la escuela como una organización de paso que no imprime un sello en sus vidas y de la cual los estudiantes esperan salir lo antes posible.

A1: No sé, es que ya estuvimos ocho años acá, ya no se me ocurre nada.

A3: No sé, yo no participo mucho.

A2: No sé, yo tampoco me imagino qué podríamos hacer.

A3: ... además nosotros ya nos vamos de esta escuela y no queremos tener problemas con nadie (Entrevista $\mathrm{N}^{\mathrm{o}} 2$, Escuela 3, Bajo ICE).

Emocionalidad (positiva o negativa). Esta categoría hace alusión a factores afectivos implicados en la explicación que los estudiantes dan acerca de su participación. Esto quiere decir que en la medida en que los estudiantes se sienten escuchados y valorados surgen sentimientos positivos de satisfacción y entusiasmo.

A2: Desde que se empezaron a hacer las actividades las cosas empiezan a cambiar, los niños se vuelven más amigables, se sienten mejor en la escuela, ya no pelean tanto, incluso de repente juegan todos (Entrevista $N^{\circ}$ 1, Escuela 3, Bajo ICE).

En cambio, cuando los estudiantes se sienten poco escuchados y no perciben cambios en su escuela, surgen emociones de frustración y se instala un sentimiento de apatía y desgano que imposibilita el encuentro con otro. Por ejemplo:

E: Ustedes... reflejaron su malestar en la escuela con las fotos del laboratorio que ustedes decían que no se estaba usando, ¿eso se mantiene? ¿Les sigue causando malestar?

A2: Sí molesta porque todavía no se puede ocupar, lo ocupa la tía Berta no más.

A3: Sí po, nos queda menos de un mes pa' salir y ni siquiera lo ocupamos una vez. Eso nos da rabia, porque igual lleva más de un año y no pasa nada (Entrevista $\mathrm{N}^{\mathrm{o}}$ 2, Escuela 3, Bajo ICE).

La presencia de sentimientos negativos se ve incrementada cuando los estudiantes se sienten, además, amenazados con posibles castigos:

A1: No hemos dicho nada, porque acá en la escuela no se puede reclamar por nada, se enojan si uno reclama.

A2: Si uno reclama después te quitan otras cosas, no nos dejan entrar a computación por ejemplo, por eso uno no se puede quejar, no conviene (Entrevista $\mathrm{N}^{\circ}$ 2, Escuela 3, Bajo ICE).

Limitaciones a la participación política. Esta última categoría alude a las limitaciones y manifestaciones de temor de los profesores y equipos directivos por la participación estudiantil de carácter político. La palabra toma despierta amenazas en el cuerpo de profesores. La toma es la 
ocupación indefinida de un establecimiento educacional por parte de los estudiantes, con la imposibilitación total de realizar actividades académicas. La toma persigue movilizar el sistema político y educativo en pos de cambios.

...sí, la verdad yo creo que todavía hay profesores y adultos que no confían en nosotros porque igual de repente nos han llegado con que tienen ciertos problemas con nuestras propuestas y también hay personas que creen que podemos llegar a tomarnos la escuela (Entrevista № 3, Escuela 1, Alto ICE).

Yo sabía que al principio no fue bien visto que los estudiantes se organizaran dentro del colegio para armar el centro de estudiantes, también sé que ciertos adultos de acá del colegio sintieron que esto era muy amenazante... porque quizás se quería ir a toma, a protestas... (Entrevista $\mathrm{N}^{\mathrm{o}}$ 2, Escuela 2, Alto ICE).

Analizando la participación estudiantil, es posible sostener que los consejos escolares, los centros de estudiantes y los reglamentos de convivencia se han orientado a generar condiciones de participación instrumental. Lo que la escuela busca a través de estas oportunidades de participación es cumplir con lo estipulado en la ley. De allí que los estudiantes sean invitados para aquellas actividades que los profesores y directivos desean instalar; y sea limitada su participación en aquellos eventos que despiertan amenazas en el cuerpo docente. La participación estudiantil al interior de la escuela adquiere una forma de control y tutela, mientras que en espacios externos a ella, los estudiantes chilenos desarrollan repertorios de participación no convencionales, como las tomas, las marchas, las protestas, etc. (Hedtke \& Zimenkova, 2013).

Convergencias y divergencias entre escuelas con buena y mala convivencia

$\mathrm{Al}$ analizar el comportamiento de estas categorías, según el ICE de cada escuela, se pudo identificar elementos que permiten ampliar el análisis. En la tabla 3 se presenta la sistematización del sistema de categorías en función del ICE de cada escuela.

\section{Discusión}

Los estudiantes dan cuenta de la existencia de espacios de participación en las cuatro escuelas participantes. Estos espacios se caracterizan por ser propuestos y regulados desde los profesores y directivos, y orientados a la resolución de problemas concretos, en que se observa una participación tutelada por adultos e instrumental.

Tabla 3

Categorías comunes y diferenciales para participación en escuelas con alto y bajo ICE

\begin{tabular}{|c|c|c|}
\hline \multirow{2}{*}{$\begin{array}{l}\text { Categorías comunes } \\
\text { Escuelas con alto y bajo ICE }\end{array}$} & \multicolumn{2}{|c|}{ Categorías diferenciales } \\
\hline & Escuelas con alto ICE & Escuelas con bajo ICE \\
\hline \multirow[t]{2}{*}{ Participación tutelada instrumental } & Sentido de comunidad & $\begin{array}{l}\text { (Falta de) Sentido de } \\
\text { comunidad }\end{array}$ \\
\hline & $\begin{array}{l}\text { Limitación a la } \\
\text { participación política }\end{array}$ & $\begin{array}{l}\text { Emocionalidad } \\
\text { negativa }\end{array}$ \\
\hline
\end{tabular}

Nota: ICE = Índice de convivencia escolar. 
En general, los estudiantes son elegidos y son invitados a participar en propuestas diseñadas por adultos. En efecto, la política pública educativa sobre consejos escolares, proyectos educativos y reglamentos de convivencia ha impuesto la necesidad de participación de los estudiantes y apoderados en la construcción del proyecto educativo (Mineduc, 2005, 2015; Unesco, 2013). La necesidad de participación, más que interpretarse como la experiencia de desarrollar competencias y habilidades que permitan a los estudiantes expresar su propia voz, escuchar a otros, integrarse, cohesionarse, practicar la democracia, etc., viene realizada a modo de cumplir con un reglamento o un indicador (Mena, Becerra, \& Castro, 2011). En todas las escuelas en estudio, donde los estudiantes son invitados o elegidos a participar, estos terminan por "aburrirse" o por "abandonar" las actividades que habían iniciado. De acuerdo con Obando Castillo (2008) y Muñoz (2011), este tipo de participación es interpretada por los estudiantes solo como un trámite.

La falta de consideración de las necesidades de los estudiantes y la rutinización de los procesos termina por aburrir al alumnado y por cansar al profesorado, que debe poner energías especiales para que las actividades propuestas no desaparezcan. Así, la participación se caracteriza por ser normativa, heterónoma (siguen los intereses de profesores), selectiva (solo para algunos estudiantes) e inestable. Es bajo esta forma de participación que interpretamos que empieza a emerger una subjetividad pasiva y dependiente. Los estudiantes esperan que los profesores les organicen distintas actividades y que sean otros quienes les realicen sus necesidades y deseos. Si bien los estudiantes hablan de participación, de acuerdo con Hart (1993), estaríamos frente a una pseudo participación estudiantil. Es decir, la escuela crea una forma que aparece como participativa, cuando en el fondo no existen espacios ni voluntades para la participación de los estudiantes.

Las escuelas que presentan una alta calidad de la convivencia escolar se caracterizan por presentar un mayor sentido de comunidad y una emocionalidad positiva. La relación entre participación y sentido de comunidad ya ha sido trabajada por Prieto (2001), quien concibe la participación como un proceso de comunicación, ejecución y toma de decisiones que permite el encuentro con un otro. Las características de esta participación toman como metáfora la idea de la familia, dejando a los estudiantes atrapados en una subjetividad infantil. Los significantes que los estudiantes utilizan son "familia" para referirse a la escuela y "mamá" para referirse a la profesora: "... somos como una familia que se lleva bien y ella nos trata bien como si fuera nuestra mamá" (Entrevista $\mathrm{N}^{\circ} 3$, Escuela 2, Alto ICE).

Estas escuelas se caracterizan, además, porque sus estudiantes expresan una emocionalidad positiva, en el sentido de que plantean sentirse bien en la escuela. No obstante lo anterior, tanto la participación como la convivencia presentan un sesgo instrumental. En palabras de los estudiantes, en la medida en que otros, los profesores, planifican y realizan actividades, la interacción escolar se vuelve más amigable y disminuyen las peleas: "Desde que se empezaron a hacer las actividades... los niños se vuelven más amigables,... ya no pelean tanto..." (Entrevista $N^{\circ} 1$, Escuela 3, Bajo ICE). Este tema es relevante, pues son estas mismas escuelas las que limitan la participación política de los estudiantes. Los profesores se sienten amenazados ante la sola intención de que los estudiantes se organicen libremente para conformar un centro de alumnos: "Es que también recibíamos amenazas por querer ser parte del 
centro de estudiantes" (Entrevista $\mathrm{N}^{\mathrm{o}} 3$, Escuela 2, alto ICE). Las características expuestas anteriormente nos hacen reflexionar en torno a la existencia de una concepción disciplinar de convivencia escolar. Se entiende la convivencia como ausencia de conflicto y peleas, en lo que DíazAguado (2005) ha denominado "perspectiva reducida" de la convivencia escolar, cuyo foco, más que estar puesto en el aprender a vivir en la diversidad y en vínculo con las oportunidades de aprendizaje, está puesto en el control, el disciplinamiento y la ausencia de conflicto.

A diferencia de estas escuelas, aquellos estudiantes que pertenecen a organizaciones educativas con baja calidad de la convivencia escolar se caracterizan por hacer explícita la ausencia de pertenencia a una comunidad educativa. Es más, se interpreta la escuela como una organización de paso que no imprime un sello en sus vidas y de la cual los estudiantes esperan salir lo antes posible. Cuando los estudiantes se sienten poco escuchados y no perciben cambios en su escuela, surgen emociones de frustración y se instala un sentimiento de apatía y desgano que imposibilita el encuentro con otro. Esto es lo que ocurre en las escuelas que presentan una baja calidad de la convivencia escolar. Vemos que tras el rechazo de participar se esconde un sentimiento de resignación (Ascorra, 2009). De acuerdo a Martín-Baró (1998) este sentimiento sería una producción social de emocionalidad que termina por transformar a los sujetos en entes pasivos que no expresan sus opiniones, no hacen respetar sus derechos y no participan de la vida política. En otras palabras, no escuchar las demandas del estudiante es no concebirlo como un sujeto con derechos al interior del espacio educativo.

A la aceptación de imposibilidad de cambio, Martín-Baró (1998) la denominó "desesperanza aprendida". Es precisamente instalar una competencia, una creencia y una emocionalidad que les comunica a los estudiantes que ellos no valen y que hagan lo que hagan, la situación no va a cambiar.

\section{Conclusiones}

Dado que el Ministerio de Educación de Chile promueve una política de participación que se traslapa con la política de convivencia escolar, el presente estudio tuvo por objetivo comprender la participación en escuelas con alta y con baja calidad de la convivencia escolar. El supuesto que se planteó fue que las escuelas con mejor convivencia escolar desarrollarían -además- una mayor participación.

Tras realizar los análisis pertinentes, se observó que todas las escuelas en estudio, sin excepción, exhibieron una participación tutelada, instrumental, heteronormativa, selectiva e inestable en el tiempo. Esto quiere decir que los estudiantes son invitados a participar por adultos de la escuela, donde su participación se restringe a un proyecto de corta duración y que, por lo general, guarda relación con mejoras para la escuela. Entonces, estamos ante un tipo de participación tutelada, instrumental y utilitarista.

A nuestro juicio, el problema más importante que acarrea la instalación de una participación tutelada dice relación con el tipo de subjetividad estudiantil que las prácticas escolares están construyendo. Es evidente que cuando solo se invita a participar a determinados estudiantes para fines de interés de los profesores, se posiciona a los estudiantes en una subjetividad pasiva y dependiente (Ascorra, 2009, 2010; Hart, 1993, 1997). El alumno está llamado a cumplir órdenes y los profesores a ejercer control (Conderman, Walker, Neto, \& Kackar-Cam, 2013). Giroux y McLaren 
(1998) señalan que el carácter transformador de la escuela viene dado por la búsqueda del "ejercicio del poder y la autoconstitución del estudiante como un sujeto activo política y moralmente" (p. 113). Pues bien, las formas de participación relatadas por los estudiantes no dan cuenta de la construcción de condiciones de posibilidad u oportunidades para una participación activa y con sentido de ciudadanía (Arnstein, 1969; Hart, 1993, 1997).

La relación entre buena convivencia y participación estudiantil se vio aún más tensionada cuando los estudiantes señalaron la prohibición que ejercen escuelas con una buena convivencia escolar ante la participación política. En este sentido, la participación en el sistema educacional chileno se encuentra en una paradoja. Mientras los profesores generan oportunidades de participación que deben orientarse hacia mejoras al interior de la escuela (participación tutelada e instrumental), los estudiantes participan de manera no convencional (toma, movilizaciones y paros) con el objeto de satisfacer demandas macroestructurales que rebasan el espacio de la escuela. La relación entre demandas internas y externas a la escuela debe ser investigada en mayor profundidad.

Los hallazgos también abren la discusión acerca del constructo índice de convivencia escolar, toda vez que, a pesar del sentido histórico y teórico del concepto convivencia, el constructo parece poder prescindir del concepto participación, tanto operacionalmente como en la práctica. Esto es coherente con las políticas educativas en Chile que han validado una participación meramente informativa y consultiva (Muñoz, 2011) y una práctica de convivencia escolar que se centra en el disciplinamiento de los estudiantes. De esta forma, si contrario a lo esperado, escuelas con alto índice de convivencia escolar presentan una participación instrumental de sus estudiantes, se torna relevante reflexionar sobre las consecuencias de promover un tipo de convivencia en que la participación promovida es mínima (el nivel más bajo de participación en la escala de Hart).

\section{Referencias}

Anderson, G. (2002). Hacia una participación auténtica: deconstruyendo los discursos de las reformas participativas en educación. En M. Narodowski, M. Nores, \& M. Andrada (Coords.), Nuevas tendencias en políticas educativas: Estado, mercado y escuela (pp. 145-200). Buenos Aires, Argentina: Granica.

Arntsein, S. (1969). A ladder of citizen participation. Journal of the American Planning Association, 35(4), 216-224.

http://dx.doi.org/10.1080/01944366908977225

Ascorra, P. \& Cáceres, P. (2002). Evaluación de los aspectos psicométricos del inventario de clima de aula "Mi Clase" (IMC). Revista Enfoques Educacionales, 3(1), 117-134.

Ascorra, P. (2009). El gerenciamiento de los estados de ánimo: estudio de caso en una organización chilena. Revista IRICE, 20, 21-32. Recuperado de https://is.gd/28jial 
Ascorra, P. (2010). Aportes de las ciencias sociales al nuevo management. Psicoperspectivas, 9(1), 1-6. Recuperado de http://www.psicoperspectivas.cl/index.php/psicoperspectivas/article/viewFile/119/384

Assaél, J., Edwards, A., López, G., \& Adduard, V. (1989). Alumnos, padres y maestros: la representación de la escuela. Estudio etnográfico en escuelas urbano-populares. Santiago, Chile: PIIE.

Bellei, C., Contreras, D., \& Valenzuela, J. P. (Eds.). (2010). Ecos de la revolución pingüina: avances, debates y silencios en la reforma educacional. Santiago, Chile: Unicef/Universidad de Chile. Recuperado de

https://is.gd/qmunKu

Bellei, C. [CIAE Uchile] (2016, 23 de mayo). A una década de la revolución pingüina [Archivo de video]. Recuperado de

https://www.youtube.com/watch?v=CwX-ZlkOHIU

Benbenishty, R. \& Astor, R. (2005). School violence in context: Culture, neighborhood, family, school and gender. New York, New York: Oxford Press.

Berg, J. \& Cornell, D. (2016). Authoritative school climate, aggression toward teachers, and teacher distress in middle school. School Psychology Quartely, 31(1), 122-139.

http://dx.doi.org/10.1037/spq0000132

Carbajal Padilla, P. (2013). Convivencia democrática en las escuelas. Apuntes para una conceptualización. Revista Iberoamericana de Evaluación Educativa, 6(2), 13-35. Recuperado de

http://www.rinace.net/riee/numeros/vol6-num2/art01.html

Carrasco, A., Bogolaski, F., Flores, C., Gutiérrez, G., \& San Martín, E. (2014). Selección de estudiantes y desigualdad educacional en Chile: ¿qué tan coactiva es la regulación que la prohíbe? Proyecto Fonide 711286. Santiago, Chile: Fondo de investigación y desarrollo en educación. Recuperado de

https://is.gd/BGOXkZ

Cohen, J,. McCabe, E., Michelli, N., \& Pickeral, T. (2009). School climate: Research, policy, practice, and teacher education. Teachers College Record, 111(1), 180-213. Recuperado de http://www.tcrecord.org/Content.asp?ContentId=15220

Conderman, G., Walker, D., Neto, J., \& Kackar-Cam, H. (2013). Student and teachers perceptions of middle school climate. The Clearing House: A Journal of Educational Strategies, Issues and Ideas, 86(5), 184-189.

http://dx.doi.org/10.1080/00098655.2013.802214

Dávila, O. (2003). Foro Nacional de Juventud México. Políticas públicas de juventud: Algunas tensiones y problematizaciones. Valparaíso, Chile: CIDPA. Recuperado de https://is.gd/RKI4iM

Dávila León, O., Ghiardo Soto, F., \& Medrano Soto, C. (2005). Los desheredados. Trayectoria de vida y nuevas condiciones juveniles. Valparaíso, Chile: CIDPA. Recuperado de http://www.cidpa.cl/wp-content/uploads/2013/05/Desheredados.pdf 
De la Fuente, L. \& Raczynski, D. (2010). Estudio de evaluación de la situación de los consejos escolares. Informe final. Santiago, Chile: Asesorías para el desarrollo. Recuperado de http://www.asesoriasparaeldesarrollo.cl/docs/315261031.pdf

Díaz-Aguado, M. J. (2005) Por qué se produce la violencia escolar y cómo prevenirla. Revista Iberoamericana de Educación, 37, 17-47. Recuperado de http://www.rieoei.org/rie37a01.htm

Espinoza, E. (2006). Impacto del maltrato en el rendimiento académico. Revista Electrónica de Investigación Psicoeducativa, 4(2), 221-238. Recuperado de http://www.investigacion-psicopedagogica.org/revista/articulos/9/espannol/Art_9_64.pdf

Instituto de Ciencia Política, Pontificia Universidad Católica de Chile. (2016). Estudio sobre participación estudiantil realizaron estudiantes de nuestro magíster para Fundación Chile. Recuperado de https://is.gd/JUTjMg

Fleet, N. (2011). Movimiento estudiantil y transformaciones sociales en Chile: una perspectiva sociológica. Polis, 10(30), 99-116. https://dx.doi.org/10.4067/S0718-65682011000300005

Giroux, H. \& McLaren, P. (1998). Sociedad, cultura y educación. Madrid, España: Miño y Dávila.

Glaser, B. \& Strauss, A. (1967). The discovery of grounded theory. Chicago, Illinois: Aldine.

Godás Otero, A., Santos Rego, M., \& Lorenzo Moledo, M. (2008). Convivir en los centros educativos. ¿Cómo lo ven los alumnos, padres y profesores? Bordón, 60(1), 41-58. Recuperado de https://dialnet.unirioja.es/descarga/articulo/2691824.pdf

Guzmán, A., Hojman, V., \& López, V. (2002). Uniones comunales de centros de padres y apoderados: una aproximación a su formación y desarrollo. En C. Bellei, V. Gubbins, \& V. López (Eds.), Participación de los centros de padres en la educación (pp. 49-65). Santiago, Chile: Unicef. Recuperado de http://www.unicef.cl/archivos_documento/30/Centro_Padres1.pdf

Hart, R. A. (1993). Children's participation: From tokenism to citizenship. Florence, Italy: Unicef, International Child Development Center.

Hart, R. (1997). Children's participation: The theory and practice of involving young citizens in community development and environmental care. New York, New York: Unicef.

Hedtke, R. \& Zimenkova, T. (2013). Education for civic and political participation: A critical approach. New York, New York: Routledge.

Heras, A., De la Riestra, M., \& Burin, D. (2010). Participación, metodologías, aprendizajes: análisis de dispositivos específicos en el marco de las políticas recientes argentinas. Psicoperspectivas, 9(1), 59-92.

http://dx.doi.org/10.5027/PSICOPERSPECTIVAS-VOL9-ISSUE1-FULLTEXT-86

Holstein, J. \& Gubrium, J. (2006). Active interview. En D. Silverman (Ed.), Interpreting qualitative data (pp.113-291). London, United Kingdom: Sage. 
Keyes, C. L. M. (1998). Social well-being. Social Psychology Quarterly, 61(2), 121-140. Recuperado de http://www.jstor.org/stable/2787065

Krause, M. (1995). La investigación cualitativa: un campo de posibilidades y desafíos. Revista Temas de Educación, 7, 19-39. Recuperado de https://investiga-aprende-2.wikispaces.com/file/view/Inv-cualitat-Krause.pdf

Ley orgánica constitucional de enseñanza (LOCE) 18.962. (1990). Recuperado de http://www.leychile.cl/Navegar?idNorma=30330

Ley régimen de jornada escolar completa 19.979. (2004). Recuperado de http://www.leychile.cl/Navegar?idNorma=232146

López, V., Ascorra, P., Bilbao, M., Oyanedel, J. C., Moya, I., \& Morales, M. (2012). El ambiente escolar incide en los resultados PISA 2009: resultados de un estudio de diseño mixto. En Centro de Estudios (Ed.), Evidencia para la política pública en educación (pp.49-94). Santiago, Chile: Ministerio de Educación, Gobierno de Chile. Recuperado de https://is.gd/PzfKBJ

López, V. \& Orpinas, P. (2012). Las escalas de agresión y victimización: validación y prevalencia en estudiantes chilenos. Revista Latinoamericana de Psicología, 44(2), 109-124. Recuperado de http://www.scielo.org.co/pdf/rlps/v44n2/v44n2a10.pdf

Martín-Baró, I. (1998). Psicología de la liberación. Madrid, España: Trotta.

Martínez, M. L., Silva, C., Morandé, M., \& Canales, L. (2010). Los jóvenes ciudadanos: reflexiones para una política de formación ciudadana juvenil. Última Década, 32, 105-118. http://dx.doi.org/10.4067/S0718-22362010000100006

Mena, I., Becerra, S., \& Castro, P. (2011). Gestión de la convivencia escolar en Chile: problemáticas, anhelos y desafíos. En J. Catalán (Ed.), Psicología educacional: proponiendo rumbos, problemáticas y aportaciones. La Serena, Chile: Editorial Universidad de La Serena.

Ministerio Secretaría General de Gobierno (MSGG). (2001). Política Nacional y transversal de participación ciudadana, período 2000-2003. Documento de trabajo. Santiago, Chile: Ministerio Secretaría General de Gobierno.

Ministerio de Educación (Mineduc). (2002). Política nacional de convivencia escolar. Santiago, Chile: Ministerio de Educación. Recuperado de http://www.convivenciaescolar.cl/

Mineduc. (2003). Política de convivencia escolar. Santiago, Chile: Ministerio de Educación. Recuperado de https://is.gd/AaPbXu

Mineduc. (2005). Decreto $N^{\circ} 24$ que aprueba reglamento de consejos escolares. Santiago, Chile.

Mineduc. (2011). Reformulación política nacional de convivencia escolar. Ministerio de Educación. Recuperado de http://www.convivenciaescolar.cl/ 
Mineduc. (2014). Fundamentos: otros indicadores de calidad educativa. Santiago, Chile: Ministerio de Educación. Unidad de Currículum y Evaluación. Recuperado de https://is.gd/gTaM16

Mineduc. (2015). Política nacional de convivencia escolar (2015-2018). Santiago, Chile: Ministerio de Educación. Recuperado de http://www.convivenciaescolar.cl/

Montecinos, C., Sisto, V., \& Ahumada, L. (2010). The construction of parents and teachers as agents for the improvement of municipal schools in Chile. Comparative Education, 46(4), 487-508.

http://dx.doi.org/10.1080/03050068.2010.519481

Muñoz, J. (2003). Análisis cualitativo de datos textuales con ATLAS/ti. Barcelona, España: Universitat Autònoma de Barcelona. Recuperado de https://is.gd/Gec7kf

Muñoz, G. (2011). La democracia y la participación en la escuela: ¿cuánto se ha avanzado desde la normativa para promover la participación escolar? Revista de Estudios y Experiencias en Educación, 10(19), 107-129. Recuperado de http://www.rexe.cl/19/pdf/196.pdf

Obando Castillo, G. (2008). La participación docente en la toma de decisiones desde la visión de la micropolítica. Educación, 17(32), 87-108. Recuperado de http://ezproxybib.pucp.edu.pe/index.php/educacion/article/view/1804

Orpinas, P. \& Frankowski, R. (2001). The Aggression Scale: A self-report measure of aggressive behavior for young adolescents. Journal of Early Adolescence, 21(1), 50-67. Recuperado de

http://jea.sagepub.com/content/21/1/50.short

Ortega-Ruiz, R. (2013, junio). Nuevas dimensiones de la convivencia escolar y juvenil: ciberconvivencia. Trabajo presentado en el V Congreso Iberoamericano de Violencia Escolar, Santiago, Chile.

Perliger, A., Canetti-Nisim, D., \& Pedahzur, A. (2006). Democratic attitudes among highschool pupils: The role played by perceptions of class climate. School Effectiveness and School Improvement, 17(1), 119-140.

http://dx.doi.org/10.1080/09243450500405217

Prieto, M. (2001). La investigación en el aula: una tarea posible. Valparaíso, Chile: Ediciones Universitarias de Valparaíso.

Reca, I. \& López, V. (2001). Participación organizada de los padres en la educación: la experiencia internacional. En C. Bellei, V. Gubbins, \& V. López (Eds.), Participación de los centros de padres en la educación (pp. 27-48). Santiago, Chile: Unicef.

Rodino, A. M. (2013). La institucionalización de la educación en derechos humanos en América Latina: avances, desafíos y una propuesta de prioridades. Sociedade e Cultura, 16(2), 257-274.

http://dx.doi.org/10.5216/sec.v16i2.32183 
Rodríguez Rodríguez, J. (2008). La participación como un acto educador y constructor de la ciudad educadora. Revista Iberoamericana de Educación, 45(2), 1-22. Recuperado de http://rieoei.org/deloslectores/1965Rodriguez.pdf

San Fabián Maroto, J. L. (2008). La voz de los estudiantes en los centros escolares. ¿Hay alguien ahí? Organización y Gestión Educativa: Revista del Fórum Europeo de Administradores de la Educación, 16(5), 27-32. Recuperado de http://fedicaria.org/miembros/fedAsturias/Apartado_9/voz_estudiantes.pdf

Schulz, W. \& Fraillon, J. (April, 2012). Students' participation in and valuing of civic engagement at school. Trabajo presentado en Annual Meetings of the American Educational Research Association, Vancouver, Canadá. Recuperado de http://research.acer.edu.au/cgi/viewcontent.cgi?article=1019\&context=civics

Thapa, A., Cohen, J., Guffey, S., \& Higgins-D'Alessandro, A. (2013). A review of school climate research. Review of Educational Research, 83(3), 357-385. http://dx.doi.org/10.3102/0034654313483907

Tonon, G. (2012). Young people's quality of life and construction of citizenship. Dordrecht, Netherlands: Springer.

Organización de las Naciones Unidas para la Educación, la Ciencia y la Cultura (Unesco, 2000). Manifiesto 2000 para una cultura de la paz y la no violencia. Recuperado de http://www3.unesco.org/manifesto2000/sp/sp_manifeste.htm

Organización de las Naciones Unidas para la Educación, la Ciencia y la Cultura (Unesco, 2013). Situación educativa de América Latina y el Caribe: hacia la educación de calidad para todos al 2015. Santiago, Chile: Salesianos Impresores. Recuperado de https://is.gd/1QbcwE

United Nations Children's Fund (Unicef, 1990). Convención sobre los Derechos del Niño. Santiago, Chile: Unicef. Recuperado de http://unicef.cl/web/convencion/

Wang M.-T. \& Degol J. L. (2016). School climate: A review of the construct, measurement, and impact on student outcomes. Educational Psychology Review, 28(2), 315-352. http://dx.doi.org/10.1007/s10648-015-9319-1

Wetzelhütter, D. \& Bacher, J. (2015). How to measure participation of pupils at school. Analysis of unfolding data based on Hart's ladder of participation. Methods, Data, Analyses, 9(1), 111-136. http://dx.doi.org/10.12758/mda.2015.004 